\title{
Classification of nasal deviation a short review
}

\begin{abstract}
Due to its central location, the nose plays a prominent role in addressing the central facial axis which helps at camouflaging a large proportion of facial asymmetry. It gives the standard norm to the facial expressions, any little nasal deviation results in conspicuous facial disharmony and is often attended by nasal airway dysfunction. The nasal deviation causes a cosmetic deformity that is often disconcerting for patients. Nasal deviation carries considerable risk at the nasal airflow by increasing the nasal valve resistance at the level of midvault and/or lower alar structures.

The lack of objective measurement of the external nasal deviation puts the professions at risk of standardizing the severity of the deviation. It deprives the nasal plastic surgeons of having a common thought about the most likely convenient and available techniques for correcting the deviation in upper, middle, and lower thirds and nasal root as the grade of deviation is indicated.
\end{abstract}

Volume II Issue I - 2019

\author{
Abdul RS Juratli \\ Consultant ENT Surgeon at Leicester University Hospitals, \\ Currently at AI Zahra Private Hospital,UAE
}

\begin{abstract}
Correspondence: Abdul Juratli, DLO-RCS Eng. FRCS (ORL-HNS) England, Consultant ENT Surgeon at Leicester UniversityHospitals 2004-20I6. Currently at AI Zahra Private Hospital, Cosmesug, Sharjah, UAE, Email juratli3@yahoo.co.uk
\end{abstract}

Received: November 04, 2018 | Published: January 04, 2019

\section{History \& legends}

History and classification: The only classification of nasal deviation in the English publishes was published by"Yong Ju Jang, MD; Jong Hwan Wang, MD; Bong-Jae Lee, MD" in 2008. on reference "Arch Otolaryngol Head Neck Surg. 2008;134(3):311-315. doi:10.1001/archoto. 2007.46". They classified the nasal deviation into 5 types: I, a straight tilted bony pyramid with a straight tilted cartilaginous vault in the opposite direction; II, a straight tilted bony pyramid with a concavely or convexly bent cartilaginous vault; III, a straight bony pyramid with a tilted cartilaginous vault; IV, a straight bony pyramid with a bent cartilaginous vault, and V, a straight tilted bony pyramid and a tilted cartilaginous dorsum in the same direction. The classification has never been used in facial plastic references due to its complexity and the lack of its clinical implication and it is an observation of seventy-five sample size with twisted noses. The classification talked about the nose as two horizontal subunits (the bony pyramid and the cartilaginous vault) concerning the facial midline which isn't identified Figure 1.

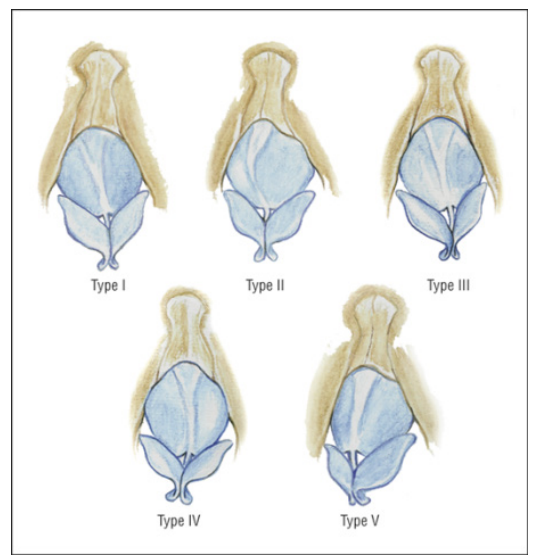

Figure I Classification of the deviated nose. For explanation of the 5 types, see the "Classification" subsection of the "Methods" section.

My study divides the external nose, for correction purposes, into three parts; upper, middle and lower. Each could be straight (Grade 0 ) or deviated first, second or third degree. It presents the nasal root separately because it is either not deviated (Grade 0), or deviated (Grade 1). The classification takes the axial facial midline and its equal distances from the brow-tip lines as the norm. ${ }^{1,2}$
The Classification helps at drawing a more precise picture of the outlining of the nasal deviation, helps at quan-tifying the improvement or the clearance of the deviation after surgery, it gives the surgeon an idea about the expected surgical time and could help at standardizing the number of the available techniques for correcting each grade of each third of the nasal deviation.

\section{Nasal parts and its aesthetic lines}

The external nose is divided into 3 parts; upper, middle and lower thirds. The upper is a bony structure made from the nasal spine of the frontal bone, two nasal bones, and the ascending or frontal process of the maxilla on both sides. The Middle third is a cartilaginous extension of the nasal dorsum consisting of a combination of triangular septal cartilage on the middle, the upper alar cartilages on each side and little sesamoid cartilages on the basal connection with the nasal bony pyriform aperture and the lower attachment of the alar base. ${ }^{3-7}$ The lower third is a fibrofatty cartilaginous structure with condensation of retaining ligaments; it is structured by the lower alar cartilages, caudal part of the nasal septum and the anterior maxillary nasal spine.

Alar base stabilization and the anterior nasal spine suture technique are essential for achieving a stable midline anchor for ensuring longterm straight nasal alignment.

The dorsal aesthetic line (DAL), provides a thoroughfare between the radix and nasal tip, it is in the middle between the two brow-tip aesthetic lines (BTAL) (Figure 2).

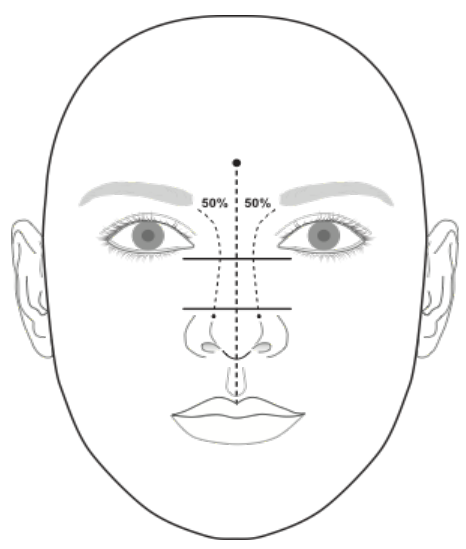

Figure 2 


\section{Brow-tip aesthetic lines}

Each line follows a slight curve from the medial aspect of the superior orbital rim at the medial brow, passing through the lateral radix and extending to the nasal tip defining points. Brow-tip aesthetic lines are expected to follow an unbroken path.

The BTALs form a subtle and fluid hourglass appearance, slightly wider at the radix and tip, with the narrow most portions in the middle third (Figure 3).

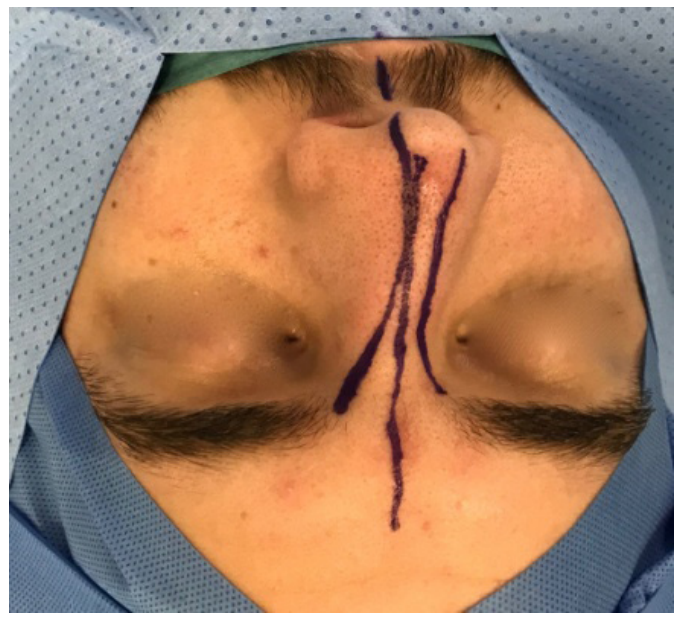

Figure 3 UIR, M2R \& L3R.

During rhinoplasty, the facial plastic surgeon must carefully recreate a smooth brow-tip contour for a natural and aesthetically pleasing result.

Assessment of the dorsal aesthetic lines is helpful for identifying dorsal irregularities that will need to be corrected. These include asymmetry, excessive width, excessive narrowness or poor definition, likewise inverted $\mathrm{V}$ deformity, which results from a disruption of the brow-tip aesthetic lines secondary to prominent caudal edges of the nasal bones and loss of mid vault support and could hide a nasal dorsal aesthetic asymmetry. ${ }^{8-12}$

\section{Deformities of the Brow-Tip Aesthetic Lines "BTALs"}

i. Irregularity: It could reflect an underlying asymmetry. Rasping, dice cartilage with Tissel glue, camouflage facial graft, adipose tissue-derived stem cells (ADSCs), or dermal fillers injection give the ideal solution as per case.

ii. Spacing: the BTALs are at equal distance from the facial axial midline, but they are more away from each other or do not fit the standard hourglass feature. We see such deformities after hump reduction without lateral osteotomies in some cases, thick nasal bones and/or thick central ethmoidal block (CEB), congenital nasal deformities, and postcentral nasal fracture. Broad and thick unilateral or bilateral spreader flap/graft could show the spacing deformity in the mid vault. Sharp pointed boxy tip as a congenital or iatrogenic deformity is another cause for the spacing deformity.

iii. Convexed deformity: Both BTALs are at equal distance from the midfacial axial line, but they are both over-projected. It reflexes over-projected nasal dorsum in the majority of cases. My study doesn't cover nasal hump deformities unless it has deviated.

iv. Concaved deformity: Both BTALs are at equal distance from the midfacial axial line, but they are under-projected either in the boney part, in the middle or the lower thirds. The former reflects under-projected nasal bony dorsum, the second reflects saddling deformity or supra-tip depression, and the lower third depression could reflect a droopy tip or a Polly-beak deformity.

v. Washed-out: When the BTALs cannot be detected in a flat nasal dorsum.

vi. Twisted Nose: When the BTALs are at unequal distances from the midfacial axial line. The deviation could be at the level of upper, middle or lower thirds and could be at 2 or 3 thirds but valiant or equal in the severity depending on the shape of the crooked nose.

\section{Classification and quantification of Nasal Deviation}

For the purpose of clinical implication, the nose is divided into three parts as I mentioned above. When the Nasal Root at the level of radix is not on the midline, it needs to be separately mentioned.

Ro: Nasal root.

U: Upper bony third.

M: Middle inner nasal valve cartilaginous third.

L: Lower external nasal valve and alar third.

Midline of the nose is identified as the vertical or sagittal line which connects between the mid-brow glabellar point and a midfiltrum point on no-congenitally deformed upper lip.

\begin{tabular}{lllll}
\hline & Nasal root & Upper third & Middle third & Lower third \\
\hline Grade 0 & Ro & U 0 R/L & MOR/L & L OR/L \\
Grade I & RoI & U I R/L & $M$ I R/L & L I R/L \\
Grade II & N/A & U 2R/L & $M 2 R / L$ & $L 2 R / L$ \\
Grade III & N/A & U $3 R / L$ & $M 3 R / L$ & $L 3 R / L$
\end{tabular}

Brow-Tip Aesthetic line: There are two lines, each one is a curvilinear line which extends from the medial aspect of the brow at the level of the medial border of the upper orbital margin extending down the lateral aspect of the radix and the dorsal projection of lateral nasal sidewall to the tip defining point at the side of the line. ${ }^{13}$

Grade (0) nasal deviation: each BTAL is $50 \%$ away from the midline (Figure 3).

Grade (1) nasal deviation: the BTALs are unequally away from the midline but none of them touching the midline. (Figure 4).

Grade (2) nasal deviation: One of the BTALs touching the axial facial midline (Figure 5).

Grade (3) nasal deviation: One of the BTALs is passing over and crossing the axial facial midline (Figure 6).

The classification is applied for pre and post op assessment and for the nose which has a history of any surgery. We need to mention any irregularity alongside the brow-tip lines separately.

The classification is applied when both BTLs touch each other in such case of having a sharp, edgy nasal dorsum, but there will be either Grade 0 when the midline meets the effused BTLs or Grade 3 when the midline does not touch the area of effusion. ${ }^{14}$ 


\section{Examples of the final classification}

Ro 1 R: The BTALs at the level of the root of the nose of the nose are at the unequal distance from the axial midline, but none are touching the midline, and the deviation is on the right, Figure 4.

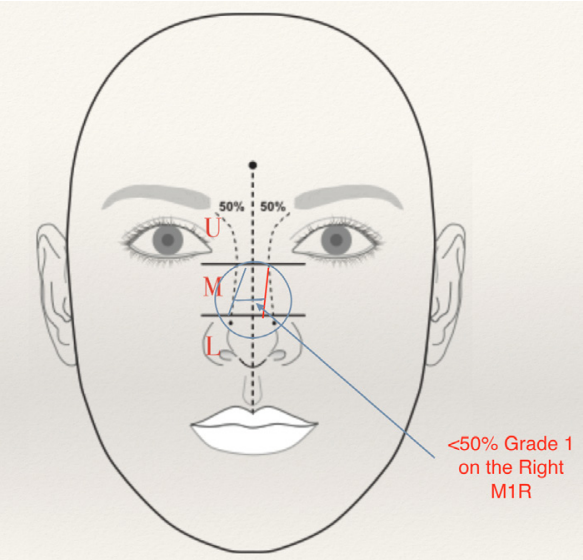

Figure 4

U 2 R: One of the BTALs is touching the midline in the upper third, and the deviation is on the right, Figure 5.
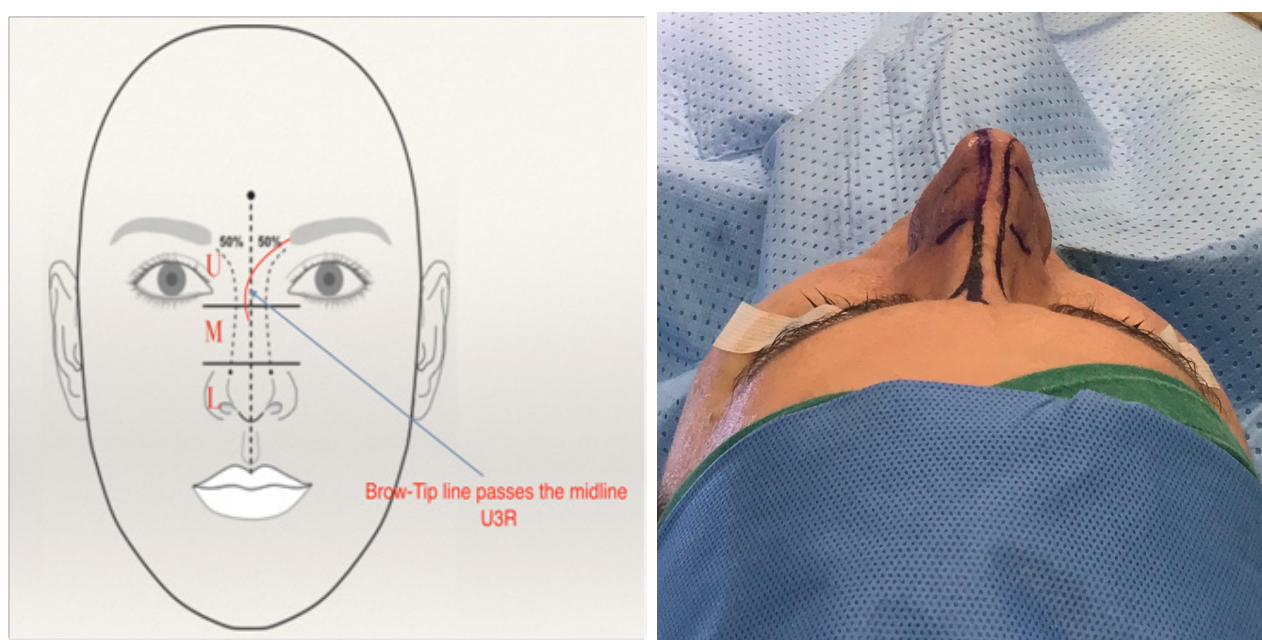

Figure 6 Twisted Nose: RoIR, U2R, M3R, L3R.
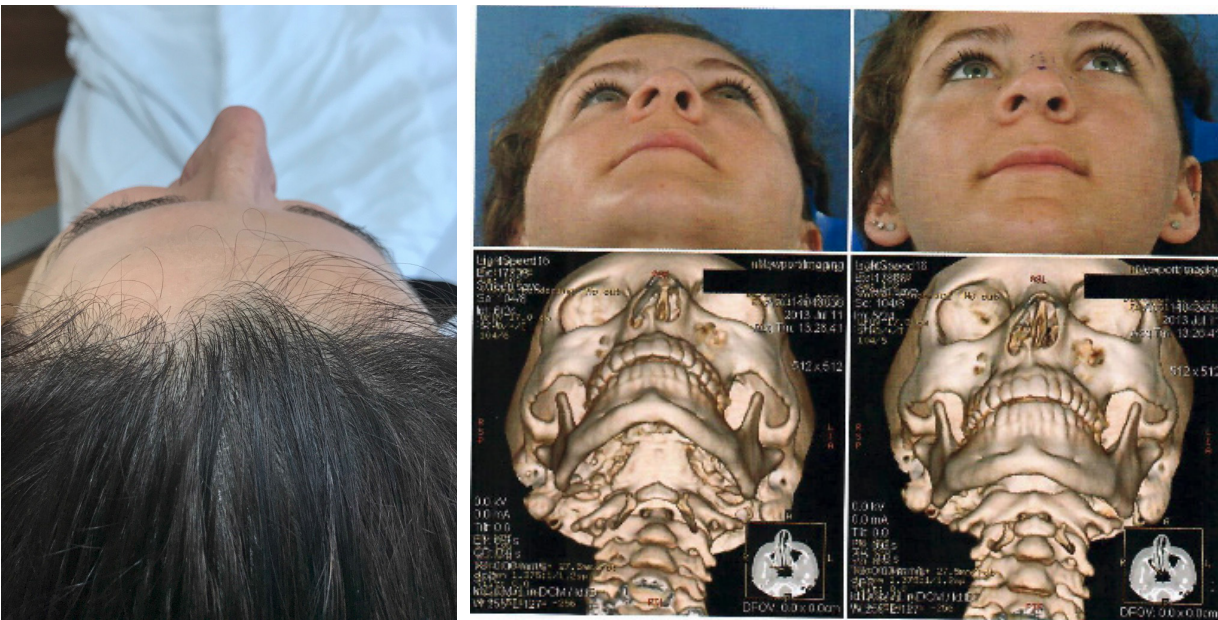

Figure $7 \& 8$ 


\section{Surgical implication for the New Classification General considerations}

Root deviation (Ro): Consider root transverse osteotomy and fracturing of the frontal nasal spine.

Upper third deviation: Consider tilted hump reduction and central ethmoidal block deviation. Medial, medial oblique, lateral, intermedial and transverse osteotomies are required depending on the severity of the deviation. Camouflage nasal dorsum and/or the short concaved side-wall could be necessarily required.

Middle third deviation: Consider thick, thin, unilateral, bilateral or extended spreader graft. Spreader flap is often applied when there is a hump reduction for $3 \mathrm{~mm}$ hight or more. Clocking suture helps in some cases, and crisscross with or without camouflaging the key-stone junction with diced cartilage and platelets poor plasma (PPP) or Tisseel glue or fascia or perichondrium grafts should be accounted to avoid a gap depression at the bony-cartilaginous junction. Extracorporeal septoplasty could be the only option to correct a severe deviation in the bony-cartilaginous junction and mid vault of the nose.

Lower third deviation: Consider stabilizing the caudal part of the septum on the midline. There are few techniques to secure the caudal septum on the anterior nasal spine. Creating straight caudal septal cartilage usually proceed the stabilization, by getting a firm L-Strut, extended septal graft, extended spreader graft or deploying $0.15 \mathrm{~mm}$ thickness, perforated PDS foil plate for temporary support. ${ }^{15-20}$

Each of the lower alar cartilage domal angle and interdomal angle should be optimized and stabilized with the support of columellar strut, tongue-in-groove or anterior septal angle suspension suture on the midline. The lateral alar crurae should be wide enough, well supported and symmetrical before the final closure.

\section{Tailoring considerations}

Quantification of nasal deviation by giving ascending numbers for each third of external nasal alignment will draw a clear picture for the listener to understand the description of nasal deviation. Specific shapes raise a risk on keystone area and suspicion about Lateral Wall Insufficiency "LWI" and nasal valve deficiency.

How the classifications help in managing cases and planning for the most convenient approach?

Ro1R or L: Transverse root osteotomy and facial asymmetry in case of non-traumatic history, are thought about, and the theory of Developmental nasal deviation become more to consider.

U1R or L: Asymmetrical hump reduction and lateral osteotomies, are considered as sufficient.

U3R or L: Raises the plan for an on-lay camouflage graft on the concave part of the bony sidewall as well as the osteotomies as per U1 including median and medial oblique osteotomy.

M1: On its own, unilateral spreader graft is usually adequate when a valve function issue is involved.

M3: Consider unilateral spreader graft, camouflage graft, asymmetrical auto spreader flap, and Clock suture at the same time. Keystone area is at significant risk, and extracorporeal septoplasty or Anterior Septal Reconstruction ASR is a technique which is, thought about in case of dorsal and caudal septal deformity. We could often find ourselves dealing with Zone 1 lateral wall insufficiency LWI.

L1: With straight and robust caudal septum, consider symmetrical intradermal followed by tip suspension on the anterior septal angle or Tongue-in-groove.

L3: with tip ptosis, asymmetrical lateral crural steal sutures and tip suspension with or without columellar strut and Shield graft or diced cartilage in glue and fascial graft should be considered dependant on the skin thickness and the severity of the ptotic tip. In case of over projected tip and tip ptosis, consider asymmetrical lateral crura overlap and vertical cut.

Finally: When the situation is Grade 2 in any of the thirds, address something in between as per the third neighbor deformity.

\section{Important consideration}

The anterior nasal valve integrity is often at risk, supporting the boundaries of the internal and external parts of the valve will give proper extended lasting nasal function. Most of the techniques as mentioned above will certainly help.

The Developmental Deviated Nose (DDN) often carries alar base level asymmetry which could require grafting the alar base-pyriform fossa junction with allogenous, homogenous or autogenous grafts at the receded side of the alar base.

\section{Acknowledgements}

The authors appreciate the assistance of all children who participated in this work.

\section{Conflict of interest}

Author declares that there is no conflict of interest.

\section{References}

1. Abdul Juratli. Twisted Nose and Anterior Nasal Valve Plasty Classification You Can Rely On. J Otolaryngol ENT Res. 2017;7(2):00200.

2. Kridel RW, Kwak ES, Watson JB. Columellar Aesthetics in Open Rhinoplasty. Facial Plastic Surgery. 2016;32(4):333-338.

3. Moubayed SP, Most SP. Correction Deviation of the Lower Third of the Nose. Facial Plast Surg. 2017;33(2):157-161.

4. Toriumi DM. Dorsal Augmentation Using Autologous Costal Cartilage or 6- Microfat-Infused Soft Tissue Augmentation. Facial Plast Surg. 2017;33(2):162-178.

5. Krane NA, Markey JD, Moneta LB. Aesthetiics of the nasal dorsum: proportions, light, and shadow. Facial Plast Surg. 2017;33(2):120-124.

6. Recker C, Hamilton GS. Evaluation of the Patient with Nasal Obstruction. Facial Plast Surg. 2016;32(1):3-8.

7. DeRosa, J. Does the Nose Have a Function Beyond Breathing? Facial Plat Surg. 2016:32(1);9-16.

8. Bilen BT, Tenekeci G. Nasal dorsum management using fragmented cartilage grafts. J craniofac Surg. 2016;27(7):1647-1651.

9. Aaron MK. Rhinoplasty: The Asymmetric Crooked Nose an Overview. Facial Plast Surg. 2016;32(1):361-373.

10. Kutubize A. Nasal aesthetic lines and rhinoplastytechnical tricks. Plast Aesthet Res. 2015;2:315-319.

11. Davis R. Rhinoplasty and concepts of facial beauty. Facial Plast Surg. 2006;22(3):198-203. 
12. Azizzadeh B, Rielly M. Dorsal hump reduction and osteotomies. Clin Plast Surg. 2016;43(1):47-58.

13. Roostaeian J, Unger JG, Lee MR, Geissler P, Rohrich RJ. Reconstitution of the nasal dor-sum following component dorsal reduction in primary rhinoplasty. Plast Reconstr Surg. 2014;133(3):509-518.

14. Sharafi M, Jalessi M, Adamson P. Newly designed upper lateral cartilage flap for pre-venting depression of the keystone area in large-nose septorhinoplasty. JAMA Facial Plast Surg. 2015;17(6):399-404.

15. Azizzadeh B, Mashkevich G. Middle Estern Rhinoplasty. In: Azizzadeh B, editor. Master Techniques in Rhinoplasty. Philadelphia. PA: Elsevier/ Saunders: 2011:387-396.

16. Cobo R, Numa W. Mestizo Rhinoplasty. In: Azizzadeh B, editor. Master Techniques in Rhinoplasty. Philadelphia, PA: Elsevier/Saunders: 2011:397-414.
17. Toriumi W. Innovative Surgical Management of Crooked Nose in Rhinoplasty. In: The Dallas Rhinoplasty Symposium Text. ST. Louis, MO: Quality Medical Publishing; 2001.

18. Daniel PK. The nasal tip: anatomy and aesthetics. Plastic Reconstructive Surg. 1992;899(2):216-224.

19. Daniel RK. Mastering Rhinoplasty: A Comprehensive Atlas of Surgical Techniques. Stuttgart: Springer; 2011:72.

20. Gordon CR, Alghoul M, Golberg JS, et al. Diced cartilage graft wrapped in AlloDerm for dorsal nasal augmentation. J Craniofac Surg. 2011;22(4):1196-1199. 